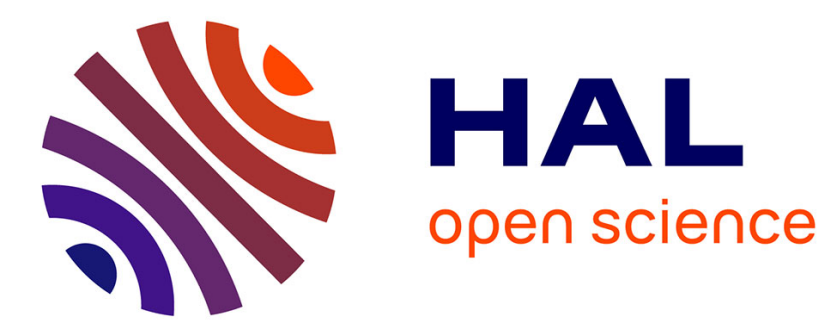

\title{
De la théorie des affects à celle des neurosciences : la perception du moi
}

\author{
Karine Bréhaux
}

\section{To cite this version:}

Karine Bréhaux. De la théorie des affects à celle des neurosciences : la perception du moi. Bulletin d'histoire et d'épistémologie des sciences de la vie , 2017, Volume 24 (1), pp.57. 10.3917/bhesv.241.0057 . hal-03006589

\section{HAL Id: hal-03006589 \\ https://hal.science/hal-03006589}

Submitted on 8 Dec 2020

HAL is a multi-disciplinary open access archive for the deposit and dissemination of scientific research documents, whether they are published or not. The documents may come from teaching and research institutions in France or abroad, or from public or private research centers.
L'archive ouverte pluridisciplinaire HAL, est destinée au dépôt et à la diffusion de documents scientifiques de niveau recherche, publiés ou non, émanant des établissements d'enseignement et de recherche français ou étrangers, des laboratoires publics ou privés. 


\title{
DE LA THÉORIE DES AFFECTS À CELLE DES NEUROSCIENCES : LA PERCEPTION DU MOI
}

\author{
Karine Bréhaux
}

Éditions Kimé | «Bulletin d’histoire et d’épistémologie des sciences de la vie » 2017/1 Volume 24 | pages 57 à 74

ISSN 1279-7243

ISBN 9782841747979

Article disponible en ligne à l'adresse :

https://www.cairn.info/revue-bulletin-d-histoire-et-d-epistemologie-des-sciences-de-lavie-2017-1-page-57.htm

Distribution électronique Cairn.info pour Éditions Kimé.

(C) Éditions Kimé. Tous droits réservés pour tous pays.

La reproduction ou représentation de cet article, notamment par photocopie, n'est autorisée que dans les limites des conditions générales d'utilisation du site ou, le cas échéant, des conditions générales de la licence souscrite par votre établissement. Toute autre reproduction ou représentation, en tout ou partie, sous quelque forme et de quelque manière que ce soit, est interdite sauf accord préalable et écrit de l'éditeur, en dehors des cas prévus par la législation en vigueur en France. Il est précisé que son stockage dans une base de données est également interdit. 
Bull. Hist. Épistém. Sci. Vie, 2017, 24, (1), 57-74

De la théorie des affects à celle des neurosciences : la perception du moi

\begin{abstract}
Karine Bréhaux*
RÉSUMÉ. Selon la traduction de Charles Apuhn, deux termes définissent l'affect: l'affectus et l'affectio. Il y a à la fois l'affecté, l'affectant et l'affection : l'affection rapportée au corps, lorsque celui-ci est affecté par un objet extérieur à lui, et l'idée d'affection que se fait l'âme de l'objet extérieur à lui et l'objet extérieur en tant qu'affectant le corps. L'affection est donc l'image que se représente l'âme, et l'affect est l'affection et l'idée de cette affection. L'affect est à la fois physiologique et psychique "agissant » (en langage philosophique moderne) sur l'âme et le corps. Les affects nous aident à construire nos propres repères. À travers l'interprétation du sentiment de la tristesse par René Descartes et Baruch de Spinoza, nous interrogerons le caractère contemporain de l'explication mécaniste des affects dans la constitution du sujet, être tiraillé par deux aspects binaires : le positif et le négatif.
\end{abstract}

ABSTRACT. According to Charles Apuhn's translation, two terms define the affect: affectus and affectio. There is at the same time the affected, the affecting, and the affection: the affection related to the body, when it is affected by an object external to it, and the idea of affection which is effected, Soul of the object outside it and the outer object as affecting the body. Affection is therefore the image which the soul represents, and affection is the affection and idea of this affection. The affect is both physiological and psychic " acting " (in modern philosophical language) on the soul and the body. Affects help us build our own benchmarks. Through the interpretation of the feeling of sadness by René Descartes and Baruch de Spinoza, we will question the contemporary character of the mechanistic explanation of affects in the constitution of the subject, being torn by two binary aspects: the positive and the negative.

* Docteur de science politique mention pensée politique et DEA de Philosophie. karinebrehaux@orange.fr. 


\section{INTRODUCTION}

Poser l'interrogation du mal-être de l'homme, cette négation avec soi-même «Pourquoi je me sens mal» ou «pas bien », revient à se plonger au cœur de l'affect, des passions. L'imagination a pour rôle de forger l'image de l'objet extérieur, une fois réalisée cette image ou idée inadéquate complète l'ensemble d'idées de l'âme (J'entends par idée un concept de l'âme, que l'âme forme pour ce qu'elle est chose pensante. Spinoza, L'Éthique, Garnier Flammarion, Paris, 1965, page $\left.69^{1}\right)$. L'affectivité renvoie à la variation de la puissance d'agir du corps sensible à la nature de rencontres extérieures. À travers l'interprétation du sentiment de la tristesse par René Descartes et Baruch de Spinoza, nous interrogerons le caractère contemporain de l'explication mécaniste des affects dans la constitution du sujet, être tiraillé par deux aspects binaires : le positif et le négatif. De la théorie des affects propre à Spinoza ou Descartes à celles développées par les neurosciences, la perception interne du moi reste mystérieuse. Comment parvenons-nous à nous représenter nous-mêmes comme être pensant ? De la mécanique des passions aux théories interactionnistes et neurocognitives, la construction de cette perception interne est en développement constant grâce au progrès technique, mais conserve des caractéristiques communes à travers les époques modernes et postmodernes.

Ici notre problématique repose sur l'interrogation à saisir la contemporanéité des explications mécanistes cartésiennes et spinozistes comme des éléments explicatifs et constitutifs de théories neuroscientifiques.

De manière historique (de la Modernité à la postmodernité), nous montrons l'absence de rupture épistémologique entre la mécanique des passions définie par Descartes et Spinoza et la controverse actuelle autour de la théorie des neurones-miroirs. L'intérêt est de démontrer le substrat philosophique et mécanique moderne nourrissant cette controverse scientifique de l'intersubjectivité en lien avec une explication neuroscientifique de nos représentations.

\footnotetext{
${ }^{1}$ B. Spinoza ${ }_{2}$ L'Éthique, Garnier Flammarion, Paris, 1965, p. 69.
} 


\section{LES AFFECTS COMME MÉCANISMES DE CONSTRUCTION DE NOTRE CONSCIENCE DE SOI.}

Les mœurs, les coutumes et le comportement des individus sont des données variables. On peut les constater, les analyser voire les observer, mais il est néanmoins difficile de les prévoir. On peut faire des approximations, élaborer des normes, mais il existe une variable que l'on ne peut ignorer, c'est la volonté individuelle, c'est ce moi qui décide d'agir de telle ou telle façon, de transgresser ou pas les règles et les lois établies, pour des raisons diverses et variées. Il nous est plus que difficile de savoir comment les individus vont agir face à des situations complexes. Face à un choix, ce n'est pas toujours la raison qui l'emporte ; les passions, si bien expliquées par René Descartes, les sentiments et les événements peuvent prendre le dessus. Ce qui fait de nous des êtres uniques et singuliers, des individus à part entière, c'est cette volonté qui nous meut. Cette recherche de constantes, de normes, d'éléments rationnels est nécessaire et propre à toute recherche, à toute analyse tenant à comprendre une situation qui paraît échapper à toute loi ou règle, et ceci afin d'en saisir les causes et les effets. Or quel est l'élément qui est à la fois propre à l'homme et permanent ? C'est le corps, sous ses différentes facettes, avec son " caractère changeant », ses états multiples, tantôt jeune, tantôt vieillissant, tantôt malade - soumis à la temporalité de notre condition humaine. Retenons pour l'instant que faire du corps l'élément central permettant d'expliquer l'homme, ainsi que sa place et son rôle au sein de la société, implique l'intervention de "spécialistes du corps humain » qui conseillent et prennent des décisions pour et parfois à la place des citoyen(ne)s. Ce glissement entre science et politique a bien évidemment des conséquences en ce qui concerne la relation à Autrui : l'Autre ne se définit pas par ce qu'il peut dire ou même faire, mais par une certaine constitution physique dont il faudra souligner la nature, et qui le place soit dans un ensemble de normes biologiques, psychologiques et de valeurs sociales, culturelles et peut-être aussi morales plus ou moins définies et sollicitées au cours de l'Histoire de l'Humanité2. À l'origine de notre faculté de sentir et re-sentir,

${ }^{2} \mathrm{~K}$. Bréhaux, in Res per nomen III. Référence, conscience et sujet énonciateur, Reims, Presses Universitaires de France, 2012. 
Descartes nous transmet une explication philosophique et mécanique des passions. L'affectivité comme variation de la puissance d'agir du corps est sensible aux rencontres extérieures.

Pour Descartes, la tristesse suscitant bien souvent la compassion ou la pitié, est le sentiment de l'âme suite à la perception d'un mal par le corps. Ce sentiment - que les philosophes du XVII ${ }^{\mathrm{e}}$ siècle appellent «passions »- intéresse particulièrement les professions de soin. En effet, l'approche humaniste des soins est globale. Le soignant s'intéresse au patient dans son intégralité, il n'est pas juste un malade à soigner, mais une personne dont il faut respecter les besoins. Dans ce cadre par exemple, la tristesse est écoutée et comprise, au même titre que les signes médicaux. Ce sentiment de tristesse en appelle alors d'autres comme l'empathie au cœur de la relation soignantsoigné.

La philosophie cartésienne va plus loin encore, car elle associe à cette réflexion humaine sur les passions une analyse mécanique ou physiologique de l'impact des «bonnes » ou «mauvaises » passions sur le corps dans la perception du moi :

L'âme n'est immédiatement avertie des choses qui nuisent au corps que par le sentiment qu'elle a de la douleur, lequel produit en elle premièrement la passion de la tristesse, puis suscite la haine de ce qui cause cette douleur, et en troisième lieu le désir de s'en délivrer [...] ce qui fait voir qu'elles sont toutes les cinq très utiles au regard du corps, et même que la tristesse est en quelque façon première et plus nécessaire que la joie et la haine, que l'amour, à cause qu'il importe davantage de repousser les choses qui nuisent et peuvent détruire que d'acquérir celles qui ajoutent quelque perfection sans laquelle on peut subsister,

L'autre cause est la tristesse suivie d'amour ou de joie, ou généralement de quelque cause qui fait que le cœur pousse beaucoup de sang par les artères. La tristesse y est requise, à cause, refroidissant tout le sang, elle rétrécit les pores des yeux. ${ }^{3}$

La relation soignant-soigné, investie immanquablement ce registre de la mimesis telle que l'a défini Descartes. Les relations en miroir

${ }^{3}$ R. Descartes, Les Passions de l'âme, article 137, Paris, Garnier Flammarion, 1989. 
entre le soignant et le patient sont nourries par ces représentations et sentiments de l'un vers l'Autre.

AuX PRÉMICES DE « SOI-MÊME COMME UN AUTRE » DE RICOEUR, LA VISION DE SPINOZA.

Pour Spinoza, l'âme est sensible à la capacité du corps à agir : elle souffrira donc que le corps soit immobilisé à cause d'une maladie et d'une perte d'autonomie. Cette conception de la relation du corps est d'autant plus actuelle, qu'elle rappelle l'impact d'un environnement favorable ou néfaste sur l'état psychologique de l'individu. Le lien de confiance entre le soignant et la personne souffrante repose entre autres sur des sentiments de compassion, d'empathie, de cordialité et de patience. Dans le langage spinoziste, ce sont des qualités ou des passions qui sont recherchées par la personne malade comme source d'apaisement et de bien-être. Le conatus chez Spinoza désigne l' " effort» que toute chose doit faire pour "persévérer dans son être », c'est-à-dire pour exister. L'individu recherche uniquement les sentiments - ou passions - positifs car il cherche à se préserver. La passion se situe par rapport à l'activité de l'âme, il existe un couple passion-action. La passion de l'âme est une idée d'une affection du corps par laquelle la puissance d'agir du corps est la diminution de penser de l'âme :

L'essence de l'âme est constituée par des idées adéquates et des idées inadéquates ; par suite elle s'efforce de persévérer dans son être en tant qu'elle a les unes et aussi en tant qu'elle a les autres; et cela pour une durée indéfinie. Puisque, d'ailleurs, l'âme, par les idées des affections du corps, a nécessairement conscience d'elle-même, elle a conscience de son effort ${ }^{4}$.

Spinoza ne fait ici que rappeler le principe d'équilibre liant le corps à l'état psychologique de l'individu. Si le corps est affecté par la maladie (avec la diminution de ses capacités), l'état psychologique (la prise de conscience de cette vulnérabilité) est aussi affecté entraînant une forme de déséquilibre. Ce type d'analyse est à l'origine de nombreuses théories psychologiques contemporaines (behaviorisme ou comportementalisme). L'individu a donc tendance naturellement à fuir toute mélancolie ou tristesse et rejette tout contact avec des êtres

${ }^{4}$ B. Spinoza, L'Éthique, proposition 9, Paris, Seuil, 1999, p. 219. 
ou des objets lui procurant ce type de mal-être. L'explication spinoziste du conatus nous donne encore des clés de compréhension dans ses échanges constants entre le soignant et le patient, entre le partage des émotions et la protection de soi tel que le phénomène de l'épuisement professionnel, quand une personne ne parvient plus «à donner » à l'Autre.

\section{LES REPRÉSENTATIONS DES SOIGNANTS : L'EFFET MIROIR VERSUS « JUGER L'AUTRE ».}

Reconnaitre la tristesse de l'Autre, c'est juger que cette personne est triste et renvoie alors à son propre référentiel de valeurs. Concevoir la négation d'un sujet, c'est la juger. Or, qu'implique l'acte de juger par essence ? Juger, c'est toujours juger quelqu'un ou quelque chose : cette personne semble angoissée, malade, cet objet n'est pas conçu selon les consignes prescrites. Le fait de juger nécessite donc la présence d'un agent (le sujet pensant de Descartes) et l'objet sur lequel s'exerce la réflexion (autre substance pensante ou substance étendue (objet) et dont il énonce ce qui pour lui pourrait être une vérité. Par exemple : «j'ai jugé préférable de ne pas venir » est une expression qui révèle un mobile, une raison dont le jugement est l'expression et dont la finalité est l'absence de ma présence. Événement réel qui manifeste l'antériorité d'une raison, qui a motivé un jugement, traduit une volonté de ne pas venir. Juger peut aussi signifier une activité prise entre deux actes : un acte de la raison dont le mobile est inconnu et l'acte de ne pas se rendre à cet endroit. On peut donc en apparence juger de tout, avoir un parti pris sur chaque événement, élément qui nous entoure, ou de rien "être neutre» «n'avoir aucun avis sur qui que ce soit ou quoi que ce soit». Dans l'hypothèse où nous aurions tout jugé, tout analysé, que nous reste-il comme activité réflexive ? Juger possède un caractère propre, qui ne peut exister que s'il est entretenu. Juger est inhérent à la nature humaine, activité du res cogitans se déclinant sur plusieurs modalités : analytique (un jugement est analytique lorsque le concept de son prédicat est inclus dans celui de son sujet, exemple « tout corps est étendu » est une proposition analytique, car l'extension spatiale est supposée par le concept de corps), synthétique a posteriori (les jugements synthétiques eux permettent d'accroitre la connaissance). Une proposition affirmant que tout corps a un poids, par exemple, 
rajoute au concept de corps celui de poids. Un jugement synthétique a posteriori impliquera nécessairement l'intervention d'une intuition sensible, à portée simplement subjective) synthétique a priori (un jugement synthétique a priori supposera que s'y adjoigne la forme pure de l'intuition). Si l'activité de juger est si proche de celle de penser, en est-elle réellement distincte? Le fait de ne pas pouvoir tout juger, signifie qu'on ne peut pas « embrasser tout par la pensée »? Dans un premier temps, juger c'est l'agir du sujet pensant, diversifié dans de multiples actions, en dynamique, produisant des actions/actes de jugements, dynamique de va et vient, car on peut "réviser un jugement », cette personne semble moins triste que tout à l'heure. Porter entre le positif (production d'actes de jugements) et le négatif (neutralité, ne pas produire de jugements, une personne qui n'est pas en capacité de consentir), le sujet pris dans sa propre temporalité, s'exprime sur ses deux modes : l'intériorité et l'extériorité. Sommesnous continuellement en train de juger? Cette personne même endormie semble triste, l'interprétation des signes du corps demeure possible même quand la communication verbale n'est plus. Kant dans La Critique de la Raison Pure, nous enseigne que c'est le temps en tant que forme a priori qui réalise la synthèse de cette succession de phénomènes. Le fait de juger est une tendance naturelle à connaître, à identifier et à classer les objets sensibles présents dans notre environnement.

Avons-nous «intérêt» à juger? Tout acte de jugement est-il intéressé ? Il peut vraisemblablement viser à une fin égoïste : la théorie du complot, la manipulation des esprits ? La racine latine commune entre le droit (jus) et juger (jubeo), nous porte à croire à une intentionnalité morale commune à «ce que l'on est en droit de faire ou de juger ». Sommes-nous en droit de tout juger librement, à notre guise ? Juger, c'est aussi être juste. Si juger revient à penser, que dire de cette liberté réduite de l'agir du sujet? Peut-on imposer une limite à l'activité de la pensée ? "Qu'est-ce qu'on ne peut pas juger ? » revient à " Qu'est-ce que nous ne sommes pas en droit de penser?». L'activité de juger engage celle de l'entendement, en tant que faculté de relier les représentations entre elles, de les classer, de les ordonner afin de les systématiser (principe de la tabula rasa chez Descartes). L'entendement est freiné dans sa tâche par l'incapacité de systématiser les «choses en soi » en tant que causes des phénomènes 
qui nous apparaissent. Il en reconnait l'existence sans pour autant les expliquer. L'existence des choses en soi résulte plus d'une croyance que d'une certitude objective (Spinoza les identifie comme les attributs d'une substance divine, Schopenhauer y voit l'expression de la volonté du Moi). La sensibilité fournit la matière nourrissant nos représentations sensibles ( $\mathrm{Il}$ me semble triste »). La recherche d'explication au jeu de la sensibilité sur l'entendement est multiple : le malin génie chez Kant, l'homme comme maitre et possesseur de la Nature, Deus sive Natura chez Spinoza, l'artiste défiguré de Nietzsche. C'est la Nature qui est la muse inspiratrice de l'homme, source de règles. Le sublime étant le sentiment nous rapprochant le plus de la Nature, car le sublime incarne cette posture autoréflexive de l'entendement prenant peur devant l'immensité de son ignorance (le tragique). Ce qu'on ne peut ni penser, ni juger, peut être imaginé. L'imagination transcendantale est ce qui relie la sensibilité à l'entendement (le «comme si » de la Troisième critique kantienne). Pour Fichte, l'entendement doit s'unir à son contraire (l'union du Moi et du non-Moi). L'activité de l'esprit est prise dans une temporalité biologique, le conduisant à s'adapter aux différentes connexions extérieures, aux accidents de la vie. Antonio Damasio, neurologue, qualifie le cerveau «d'émotionnel» soumis également à des changements invariants (du processus de vie à celui de la mort) et variants (les accidents pouvant créer des lésions) :

À la suite d'une lésion neurologique dans certains sites bien spécifiques de leur cerveau, les malades ont perdu une certaine catégorie d'émotions, et de manière parallèle et tout à fait considérable, ont perdu leur capacité à prendre des décisions rationnelles. ${ }^{5}$

Antonio Damasio loue l'héritage spinoziste notamment dans sa vision ontologique de l'individu :

On n'insistera jamais assez sur l'importance des faits biologiques dans le système de Spinoza. Vu à la lumière de la biologie moderne, ce système est conditionné par le fait que la préservation de la vie dépend de l'équilibre de ses fonctions et donc de la régulation de la vie; par le fait que le statut de la régulation de la vie s'exprime sous forme des

5 A. Damasio, "Le sentiment même de soi. Corps, émotions, conscience », C. Tiercelin et C. Larsonneur, Odile Jacob, 1999, p. 48-49. 
affects-la joie, la tristesse- et est modulé par les appétits, les émotions et la précarité de la vie peuvent faire l'objet d'une connaissance et d'une appréciation de l'individu humain du fait qu'il est doté d'un soi, d'une conscience et d'une raison connaissante. ${ }^{6}$

Catherine Malabou y voit une "plasticité du cerveau », s'adaptant aux aléas de la vie, mais dont les conséquences émotionnelles sont importantes puisque destructrices de la personnalité initiale :

Or quel est ce pouvoir de transformation, ce pouvoir plastique qui conduit une personne à devenir étrangère à elle-même ? Comment caractériser ce pouvoir de changement sans rédemption, sans téléologie, sans signification autre que l'étrangeté ? Les nouvelles identités des patients neurologiques ont toutes un point commun : étant toutes affectées, à des degrés divers, par des atteintes des sites inducteurs d'émotion, elles témoignent de cette désaffection ou de cette froideur. Une absence souvent insondable. De ce fait, comment penser la désertion de la subjectivité, l'éloignement du sujet qui ne devient pas étranger à quelque chose, qui ne devient pas l'autre de quelqu'un, l'autre pour quelqu'un, mais qui devient cet apatride ontologique intransitif, sans corrélatif, sans génitif, sans patrie métaphysique de départ ? ${ }^{7}$

Cette idée d'une plasticité cérébrale destructrice défendue par Catherine Malabou nous renvoie aux théories du self, à l'évolution du Moi par rapport à l'Autre, parfois pris dans une tension contradictoire mais constructive comme le défendait préalablement Fichte dans sa théorie du moi et du non-moi.

\section{LA CONSTRUCTION DE LA NOTION DE SOI: L'APPORT DES NEUROSCIENCES COGNITIVES.}

Les neurosciences cognitives s'intéressent à la notion de soi. Le soi est à la fois un ensemble de représentations mentales personnelles et multidimensionnelles que l'on a sur nous-mêmes et le phénomène de conscience de soi qui lui est associé. Le soi est à la fois à la base de notre identité individuelle et de notre sentiment de continuité dans le

\footnotetext{
${ }^{6}$ A. Damasio, «Spinoza avait raison. Le cerveau des émotions », traduction française Jean-Luc Fiel, Odile Jacob, 2003, p. 184-185.

7 C. Malabou, Souffrance cérébrale, souffrance psychique et plasticité, Études, 2011/4, t. 414, p. 491.
} 
temps. Nous pouvons ici reprendre les propos de Jean-Claude Ameisen :

Percevoir le passage du temps, percevoir ce fleuve qui s'écoule en nous - ne bat pas au rythme d'un métronome, ne bat pas au rythme d'un chronomètre, d'une horloge mais émerge sans cesse au rythme de notre vie intérieure. Ce que nous appelons notre conscience du présent, de l'instant présent, est une oscillation permanente entre mémoire et anticipation, entre souvenirs et désirs, entre nostalgie et attente. En fonction de nos souvenirs, de nos émotions, de nos espoirs et de nos craintes, en fonction de ce que nous avons déchiffré et compris du passé, et de ce que nous imaginons de l'avenir. Temps passé et temps futur, dit TS Eliot, Ce qui aurait pu être et ce qui a été, se projettent vers une fin qui est toujours présente. ${ }^{8}$

L'existence et le rôle d'Autrui dans la construction de représentations mentales personnelles semblent essentiels. C'est pourquoi le soi apparaît en relation étroite avec la théorie de l'esprit conçue comme la capacité à inférer des représentations ou des états mentaux à Autrui. Les études d'imagerie cérébrale récentes mettent en évidence l'activation d'un réseau neuronal commun au niveau du cortex préfrontal médian et du précunéus et nous conduit à formuler le postulat suivant : notre capacité à se représenter nos pensées et celles de l'Autre semble avoir une origine cérébrale similaire.

Conscience de soi et conscience de l'Autre peuvent donc s'envisager dans une interaction bidirectionnelle à l'origine du fondement de notre identité individuelle et de la compréhension d'Autrui, assurant ainsi la régulation des comportements et le bon déroulement des interactions sociales ${ }^{9}$.

L'idée est de se construire par rapport à un Autre. Platon, dans sa théorie de la réminiscence, nous montre que notre âme se définit par rapport à des connaissances antérieures dans d'autres vies (Ménon, 81b).Ici, l'élaboration de connaissances est extérieure et réelle, puisque ce sont les individus qui, dans l'indifférenciation, nous renseignent sur nos identités. Ce courant de neuropsychologie s'inscrit

${ }^{8}$ J.-C. Ameisen, Sur les épaules de Darwin, les battements du temps, éditions Babel, 2014, p. 27.

9 C. Duval, B. Desgranges, F. Eustache, P. Piolino, Le soi à la loupe des neurosciences cognitives : de la conscience de soi à la conscience de l'Autre, Psychol NeuroPsychiatr Vieil, vol. 7, n. 1, mars 2009, p. 8. 
dans une perspective communautarienne où le moi s'identifie et se construit dans la différenciation avec l'Autre :

Outre le concept de soi, l'identité se caractérise aussi par rapport à celui d'Autrui. Oscillant entre similarité et altérité, l'identité dévoile ainsi toute sa complexité. Afin d'en réduire la difficulté théorique, nous l'assimilerons au soi (ou self en anglais). Il est en effet pertinent de concevoir le soi comme la source de la construction de l'identité voire d'un modèle d'identité de l'individu, à partir duquel il se perçoit et se définit en tant que personne unique et distincte d'autrui. ${ }^{10}$

Prendre conscience de soi, se percevoir, se décrire, se reconnaitre se fait dans nos rapports au monde extérieur, incluant d'autres individus. Prendre conscience de l'Autre, c'est le percevoir différent de nous. Constater la tristesse d'une personne c'est marquer la différenciation de tempéraments, de degrés à cet instant entre cet Autre et moi-même. Ce courant dépasse le dualisme cartésien (sujet/corps) bien que s'inscrivant dans son prolongement. Certes le sujet est dans une quête de son identité personnelle (« Qui suis-je?») mais pose aussi l'invariabilité du soi au cours du temps comme le ressenti phénoménologique d'être une seule et même personne au cours du temps, déjà défini par Heidegger dans sa distinction de l'être et de l'étant). Le soi est donc la combinaison d'une posture réflexive de juger qui « je suis » et « comment je suis ». Ce deuxième axe est plus proche de la vision spinoziste que celle de Descartes, car Spinoza défend déjà cette persistance du soi à travers le conatus : "Chaque chose, autant qu'il est en elle, s'efforce de persévérer dans son être. $»^{11}$, "L'effort par lequel toute chose tend à persévérer dans son être n'est rien de plus que l'essence actuelle de cette chose. $»^{12}$. Il est désigné par la théorie du double-aspect :

Dans la littérature, il apparait que la définition du soi - que l'on peut également employer à la première personne (le moi) reste encore floue et très morcelée. Au regard des différentes disciplines traitant du soi ou de l'identité, de leur conception ou de leur modélisation théorique, il semble ardu d'en donner une définition complète et précise. Pourtant même si les termes diffèrent d'une spécialité à une autre, on retrouve des éléments communs. Dans une démarche intégrative, on

\footnotetext{
${ }^{10}$ Idem, p. 8.

${ }^{11}$ B. Spinoza, L'Éthique, livre 3, proposition 6, Paris, Seuil, 1999, p. 217.

${ }^{12}$ Idem, livre 3, proposition 7, p. 217.
} 
peut y voir la base sous-jacente d'une réalité générale de l'identité. Ainsi, si accord unanime il y a, il repose sur la théorie du double aspect : une conception unitaire de la personne qui fait place à la dimension objective et subjective de celle-ci. Définir l'identité personnelle revient donc à prendre en compte simultanément et nécessairement deux dimensions fondamentales : d'une part celle qui permet de répondre à la question " qui suis-je ? », d'autre part, celle qui donne à expérimenter le ressenti phénoménologique d'être une seule et même personne au cours du temps. $»^{13}$.

Le caractère stable du self ne l'empêche pas de se décliner sous différentes facettes : self social, self désirable, self désiré. Cooley définit le «soi-miroir », l'Autre tel un miroir, comme le reflet de nous-mêmes. Nous assimilons les jugements d'Autrui, là où nous montrions une variation de degré dans la reconnaissance des affects de l'Autre peut aussi interférer une adaptation ou un mimétisme dans ce renvoi d'affects : l'empathie, la compassion, l'impact sur son propre comportement. Plus qu'une interaction liée notamment aux actes de jugements, nous nous nourrissons des comportements, des jugements, des affects d'Autrui :

En effet prendre conscience de soi, se percevoir, se décrire, se connaitre ou se reconnaitre se fait nécessairement par nos relations avec le monde extérieur, incluant aussi les autres individus. Prendre en compte « la conscience de l'Autre » ou plus objectivement son état mental revient à construire, créer, déduire des représentations sur les autres, distinctes de celles qui nous définissent individuellement et qui vont faire notre personnalité. ${ }^{14}$

Ce que les neuropsychologues désignent par la théorie de l'esprit nous renvoie à des travaux antérieurs philosophiques menés sur l'entendement par Kant dont son interprétation de "l'éveil de la Raison ", Descartes dans l'exercice du doute méthodique - ce qui revient à dire que le sujet est apte à dissocier les noumènes en tant que phénomènes des choses en soi -, les représentations de soi (perception intérieure, ce que je ressens) et les choses en soi (choses inaccessibles par la pensée de l'ordre de l'inconscient ou de la métaphysique) :

\footnotetext{
${ }^{13}$ C. Duval, B. Desgranges, F. Eustache, P. Piolino, op. cit.

${ }^{14}$ Idem, p. 8.
} 
Dire qu'un individu possède une théorie de l'esprit, signifie que celui-ci attribue des états mentaux à lui-même et aux autres. Un tel système d'inférence de ce genre peut être convenablement considéré comme une théorie, d'abord parce que de tels états ne sont pas directement observables et ensuite car ce système peut être utilisé pour des prédictions, notamment sur le comportement des autres. ${ }^{15}$

Selon Premack et Woodruff (1978), ce qui m'informe est ce qui me différencie d'Autrui, le fait par exemple de capter un regard triste me renseigne sur l'état d'esprit de la personne mais cette analyse empirique résulte de ma perception sensible extérieure et ne peut être interprété comme une vérité. Si bien que les signes extérieurs projetés par les corps (mécanique cartésienne) peuvent être interprétés soient comme des manifestations volontaires d'Autrui (Schopenhauer) soit comme des signes imaginaires (noumènes de Kant) soit comme l'expression de l'âme (Descartes) ou d'un affect (la tristesse chez Spinoza). Croiser les traditions philosophiques modernes à la théorie de l'esprit en neuropsychologie nous permet de démontrer qu'un affect tel que la tristesse ressenti par autrui a plusieurs sens : noumène, représentation de soi, signe du corps, signe de l'âme, représentation mentale (théorie de l'esprit). Et chaque sens a une valeur physique ou métaphysique, et multiplie les possibilités d'interprétation de la réalité de façon vraie ou erronée. Les premières correspondent aux représentations que l'on a de la réalité d'une situation, de la croyance d'un personnage (exemple: je pense que $\mathrm{X}$ pense que...); les secondes aussi appelées "méta-représentations » correspondent aux représentations internes que l'on a sur soi-même ou qu'une personne a d'une autre personne (par exemple: je pense que je suis en train de penser. Cela nous renvoie directement au modèle de compréhension de l'intersubjectivité dit « simulationniste » car reposant sur le partage des représentations des individus. Nicolas Georgieff, dans son article «Penser l'Autre, être pensé par l'Autre : intérêts de la notion de la théorie de l'esprit pour la psychopathologie », énonce ces deux courants de compréhension de l'intersubjectivité dans laquelle notre problématique s'inscrit :

${ }^{15}$ Ibid, p.13. 
Langage et action suggèrent ainsi deux modèles de compréhension de l'intersubjectivité pour les neurosciences cognitives; l'un est « inférentiel » et reste abstrait, il suppose des opérations de calcul, inductives et déductives, opérant sur des contenus mentaux traduits par le langage dans un contexte situationnel précis, et a été développé notamment par D. Sperber et D. Wilson (1989) dans leur ouvrage sur la pertinence. La seconde perspective, largement développée dans ce volume (cf. V. Gallese) est couramment nommée « simulationniste ». Neurophysiologique à l'origine, elle repose sur la notion de partage de représentations motrices entre deux individus. L'articulation entre ces approches reste à faire, ébauchée par les hypothèses encore spéculatives quant à l'origine motrice du langage et l'impact de la structure de la représentation motrice sur le langage (la syntaxe) et la pensée (Rizozolatti et Arbib, 1998). ${ }^{16}$

\section{LA CONTEMPORANÉITÉ DES EXPLICATIONS CARTÉSIENNES ET SPINOZISTES DES AFFECTS, ET PLUS EN ENCORE DE L'ESPRIT.}

L'intérêt de la notion de la théorie de l'esprit est de poser clairement dans le champ des sciences de l'esprit cette question : en quoi consiste la propriété de l'esprit qui assure la représentation de l'esprit par l'esprit? Qu'est-ce que penser l'autre et être pensé par l'autre? Comment s'organise la représentation des pensées et émotions, croyances, désirs, de l'autre? Comment une activité psychique est-elle modifiée c'est-à-dire influencée par l'autre ? Dès sa naissance, l'individu ajuste son comportement à ceux perçus dans son environnement immédiat pour des questions de survie, de sorte que des théories comportementalistes à commencer par celle de Jean Piaget confortent cette idée que nous sommes en permanence dans «le penser l'Autre», de représenter son activité mentale, sur ce décodage me permettant en retour de réguler mon discours.

PHILOSOPHIE RATIONALISTE EN ÉCHEC? COMMENT INTÉGRER DES JUGEMENTS SYNTHÉTIQUES A PRIORI COMME MODE D'EXPLICATION DE FAITS NEUROLOGIQUES?

Les caractéristiques d'une philosophie rationaliste reposent sur l'accord que la réalité spécifique d'une raison entendue s'apparente à un ordre nécessaire des idées et des choses, ou une activité spirituelle

${ }^{16}$ N. Geogieff, «Penser l'autre, être pensé par l'autre: intérêts de la notion de théorie de l'esprit pour la psychopathologie », le carnet psy, 2005/4, n. 99, p. 24. 
autonome constitutive de l'expérience. Admettre la raison vaut soit pour tout comprendre, soit du moins pour comprendre ce qui nous est accessible et pour régler tout ce qui dépend de nous. La Raison abolit ou absorbe en elle tout prétendu principe de connaissance et d'action suffisant à l'homme et se suffisant à elle-même. Revenons sur la théorie des idées claires et distinctes de Descartes :

J'appelle claire celle de la connaissance qui est présente et manifeste à un esprit attentif, de même que nous disions voir clairement les objets lorsque présents à nos yeux ils agissent assez forts sur eux et que nos yeux sont disposés à les regarder; et distincte celle qui est tellement précise et différente de toutes les autres qu'elle ne comprend en soi que ce qui parait manifestement à celui qui la considère comme il faut. ${ }^{17}$

Une idée claire peut être confuse, ce qui veut dire partiellement vraie. Ce à quoi Spinoza se référait en parlant de « choses inadéquates et confuses ». Le propre de la représentation confuse est que je ne puisse discerner exactement où commencent les limites et les vides. La vraie et formelle fausseté se borne aux jugements produits, le sentiment peut être connu d'une connaissance claire et distincte, une façon confuse de penser. Les différences de clarté font ressortir deux états d'une faculté de connaître : cette dernière peut être livrée à ellemême et voir claire, elle peut aussi être troublée par l'influence du corps et par ce que suscite ce corps d'étranger en elle à la connaissance, brouillant sa vue et troublant l'esprit. Il faut dissocier la connaissance intellectuelle de la connaissance sensible :

C'est une seule et même force que si elle applique avec l'imagination au sens commun est dite « voir » «toucher », etc. si elle s'applique à l'imagination seule en tant que celle-ci est revêtue de figures variées, est dite se souvenir ; si elle s'applique à l'imagination pour en former de nouvelles est dite « imaginer » ou « concevoir » enfin si elle agit seule est dite entendre. » (Règle 12).

Le sens commun et l'imagination désignent des parties du corps, du cerveau. La force intellectuelle agit tantôt isolément, tantôt sous l'influence du corps. Pour Descartes, l'esprit est distinct du corps et se connait lui-même avant le corps. Mais il ne faut pas se méprendre et

${ }^{17}$ R. Descartes, Les principes de la philosophie, Paris, Garnier Flammarion, 1989, p.117. 
asseoir la physique cartésienne et spinoziste à la métaphysique. Pour Spinoza, le monde découle de la notion de Dieu avec la même nécessité que les propriétés du triangle découlent de sa définition. Le créateur est transcendant et extérieur à ses créatures, les êtres ne peuvent se suffirent à eux-mêmes. La théorie cartésienne des notions primitives est le pendant de ce volet métaphysique à la physique cartésienne. En effet, l'extension de la pensée et l'union de l'âme et du corps dépendent pour la première des figures et des mouvements, et pour la seconde de la force qu'a l'âme de mouvoir le corps et la force qu'a le corps d'agir sur l'âme en causant des sensations et des passions. Tout ce qui pense est ou existe, enseigné par la perception qu'il a de la conscience de lui-même d'une liaison nécessaire entre sa pensée actuelle et son existence. Pour penser, il faut être, le doute hyperbolique s'étend à nos jugements. Les notions primitives assurent que nous ne pouvons pas les chercher ailleurs que dans notre âme. La notion de l'infini est une notion primitive, elle est la plus primitive de toutes les notions. Les limites des sciences techniques de l'époque se ressentent dans les analyses et le recours à la métaphysique adossé à la croyance est nécessaire.

LA THÉORIE DES NEURONES-MIROIRS, VERSION CONTEMPORAINE DES PASSIONS DE L'ÂME?

La théorie des neurones miroirs s'inscrit dans la défense de cette conception des «cognitions sociales». Ce que souligne Nicolas Georgieff : «La découverte des «neurones miroirs » (Rizzolatti et al, 1996) a été un argument clé en faveur de cette seconde conception des « cognitions sociales ». Elle est en effet à l'origine de l'hypothèse d'une propriété transitive ou spéculaire du cerveau permettant de générer des configurations d'activité, et donc peut-être des représentations mentales ou des états mentaux, analogues chez soi et autrui, en rapport avec le comportement moteur (des représentations d'action) et donc par extrapolation avec les représentations du but, de l'intention, de l'état émotionnel et de la croyance sur le monde en rapport avec ce comportement. $\rangle^{18}$

18 N. Geogieff, «Intérêts de la notion de théorie de l'esprit pour la psychopathologie », La psychiatrie de l'enfant, 2005/2, vol. 48, p. 348. 
Aujourd'hui, la théorie des neurones miroirs dans ses aspects cliniques, attestent l'idée d'une connexion physiologique entre le cerveau et notre faculté de ressentir et de penser. Si bien que l'idée d'un jugement synthétique a priori selon lequel la production de la connaissance de la tristesse est possible indépendamment de l'expérience en raison de son caractère intuitif est revisitable. Les recherches autour de cette théorie des neurones-miroirs initiées par Rizollatti chez le singe (Rizzolatti, Fadiga, Gallese et Fogassi, 1996) et confirmées chez l'homme, mettent en évidence que des neurones s'activent quand on réalise une action ou que l'on observe quelqu'un la réaliser. Ces neurones, dénommés neurones-miroirs, s'activent différemment non seulement selon l'action (attraper un objet) mais aussi selon l'intention qui lui est associée (attraper un verre d'eau pour le boire). Le fonctionnement des neurones-miroirs n'est pas lié à des processus d'imitation, mais consiste en une tendance de reproduire inconsciemment les comportements et les états psychologiques d'autrui. Cela proviendrait de fait que les neurones miroirs seraient liés au développement de l'empathie et apparait probable que nous percevions les émotions d'autrui en activant les mêmes émotions en nous. La constitution de la relation psychique se fait nécessairement par cette relation à autrui dont les sources neurologiques sont peut-être à trouver du côté des neurones-miroirs En langage soignant-soigné, on note une empathie naturelle et l'impact des émotions de l'un à l'autre, à la défense de la théorie des neurones-miroirs, on admet que les projections extérieures des états d'âme d'autrui ont un effet psychologique mais aussi neurologique sur moi. $\mathrm{Ce}$ modèle neurocognitif rattaché aux cognitions sociales est le fruit d'une continuité des visions mécanistes telles que Descartes et Spinoza les définissaient sous la Modernité en l'état actuel de leur progrès scientifique et technique. Les affects, expressions des sentiments, affections et ressentis face à l'Autre, constituent les expressions de ce mécanisme spéculaire et transitif des représentations du moi et d'Autrui. Comme le souligne Nicolas Georgieff :

Il est remarquable que les modèles neurocognitifs rattachent aujourd'hui les cognitions sociales, l'accès à l'Autre, à un mécanisme essentiellement spéculaire et transitif de représentations partagées ou d'activations communes « en miroir » qui insiste sur la part commune entre soi et Autrui et définit un mode de représentation ou de 
connaissance de l'Autre basé sur le partage du même. De manière paradoxale, l'accès à l'Autre semble ainsi reposer sur l'accès à soi, dans un "système du même ${ }^{19}$.

Cependant, nous éprouvons les limites de ce modèle des neurones miroirs car il n'est pas possible de déterminer l'organisation du système de partage des représentations de la psyché et de l'altérité, de différencier ce qui relève de ma propre représentation ou d'une représentation secondaire fondé à partir d'une autre ne m'appartenant peut-être pas, ce qui relève de l'expérience de ma propre expérience ou de celle de l'altérité. La clinique alliée au progrès technique des neurosciences saura peut-être un jour nous éclairer sur les représentations du soi et de l'Autre, sur l'altérité psychique, ces constituants de notre plasticité cérébrale.

\section{CONCLUSION}

Le caractère postmoderne des explications mécanistes de Spinoza et Descartes trouve écho dans le développement de théories neuropsychologiques et psychanalytiques du self. Cette filiation trouve sa reconnaissance actuelle dans l'objectivité scientifique de la perception externe sur la faculté de l'entendement. Ainsi, reconnaitre l'émotion de l'Autre m'arrache des larmes (phénomène de mimesis) mais active aussi des zones neuronales sous la volonté de l'entendement. Nous confirmons ici l'absence de rupture épistémologique entre les traditions modernes cartésiennes et spinozistes et les courants postmodernes des neurosciences. Plus qu'une absence de rupture, une réelle filiation existe entre les théories des affects de Descartes et de Spinoza et les approches défendues dans les théories du self. Ceci nous laisse penser que ce sont les substrats ontologiques issus des philosophies cartésiennes et spinozistes qui nourrissent les approches en cognitions sociales actuelles. Reste au progrès technique à nous éclairer sur la nature du partage des échanges entre nos représentations et celle d'Autrui, sur la potentialité et la plasticité de nos cerveaux.

\footnotetext{
${ }^{19} \mathrm{~N}$. Georgieff, Intérêts de la notion de théorie de l'esprit pour la psychopathologie, La psychiatrie de l'enfant, 2005/2, vol 48, p. 367.
} 